\title{
Modeling Energy Efficiency in Wireless Internet Communication
}

\author{
Enrico Rantala \\ Nokia Research Center \\ Helsinki, Finland \\ enrico-
}

henrik.rantala@nokia.com

\author{
Arto Karppanen \\ Nokia Research Center \\ Espoo, Finland
}

arto.karppanen@nokia.com seppo.granlund@nokia.com

\author{
Seppo Granlund \\ Nokia Research Center \\ Espoo, Finland
}

ABSTRACT

\begin{abstract}
For wireless mobile Internet users the length of the battery life is one of the most important performance factors. The energy efficiency of the data transmission over radio is a key component affecting the battery lifetime. This paper investigates WLAN energy consumption in network communication on a Mobile handset. We introduce an energy model that allows analysis and simulation of the energy efficiency of the Internet protocols on a Wireless Network Interface, and have extended the NS-2 simulation platform to allow investigating the energy consumption of the Radio Modem and the Power Amplifier in WLAN 802.11g network interface of a mobile device. We have also validated our model against measurements on real wireless hardware, and show that the simulation results closely match the real world behavior. We claim to present more detailed and accurate model of the WLAN energy consumption than what is done by the past work that allows designing and optimizing future Internet protocols towards more energy efficient behavior.
\end{abstract}

\section{Categories and Subject Descriptors \\ C.4 [PERFORMANCE OF SYSTEMS]: Measurement \\ techniques, Modeling techniques, and Performance attributes}

\section{General Terms}

Measurement, Performance, Experimentation

\section{Keywords}

Wireless networking, energy efficiency, TCP, network simulation

\section{INTRODUCTION}

The length of the battery life in a wireless handheld communication device is one of the most important performance criteria for a mobile user. Besides developing batteries with better energy capacity, the length of the battery life can be extended by reducing the energy consumption in a mobile device. One of the most significant activities that consume energy is transmitting data over a wireless radio interface. In this paper (see also the extended version [2]) we introduce an energy consumption model that can be used for Internet protocol energy efficiency simulations and analysis. One of the key energy consuming components related to wireless transmission is the Wireless Network Interface (WNI) that takes care of the transmission of the packets to the physical medium. We present a model for IEEE 802.11g (WLAN) WNI in which the battery current drawn by the Power Amplifier (PA) and the Radio

Copyright is held by the author/owner(s).

MobiHeld'09, August 17, 2009, Barcelona, Spain.

ACM 978-1-60558-444-7/09/08.
Modem (ASIC) are modelled separately.

The energy consumption of mobile handsets has been under extensive research, but there are not many published studies concerning detailed WNI energy consumption and simulation. According to our knowledge Stemm and Katz [4] did the first study analysing WNI energy consumption, and they presented methods that have been used in many later studies. The work by Ebert et al. [1] also has close relationship to our work, since they discuss WNI measurement, simulation model parameterization and validation. In Singh [3], the energy consumption of three TCP versions - Reno, Newreno and SACK has been compared based on measurement experiment and by emulating various network conditions.

\section{ENERGY MODEL}

Concerning the energy consumption drawn by IEEE $802.11 \mathrm{~g}$ Wireless Network Interface (WNI) i.e. WLAN modem, there are two fundamental components to deal with: the Radio Modem (ASIC, Application-Specific Integrated Circuit) and the Power Amplifier (PA). Figure 1 illustrates the WNI components that are relevant to the energy model and also the measurement system. The main role of the ASIC is to run transceiver algorithms and MAC (Medium Access Control) protocol functions. The PA amplifies transmission signal so that it can be driven to the antenna. The main task of the host CPU (Central Processing Unit) is to run applications and upper layer protocols. Modeling the energy consumption of the host CPU is left for future work.

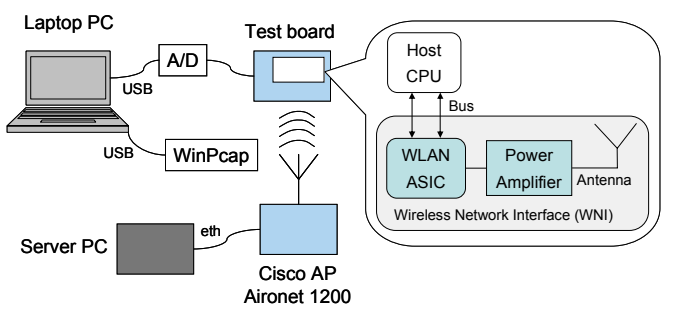

Figure 1. Schematics of the measurement system.

For the energy model we assume that the ASIC is mostly either in transmitting or receiving mode (active bulk data transfer) in which case the ASIC battery power is fairly steady throughout the experiment. This can be seen in the measurement sample in Figure 2 where transmitting periods dominate the file upload. To take into account the impact of sleep, idle, receiving and transmitting modes in a simulation, a detailed tracing capability of ASIC states is required (left for future work). The PA is highly active only on transmitting periods like shown in Figure 2. In between transmitting periods, only leakage battery current is drawn. We model the PA energy consumption per-packet basis and take also into account idle periods. 


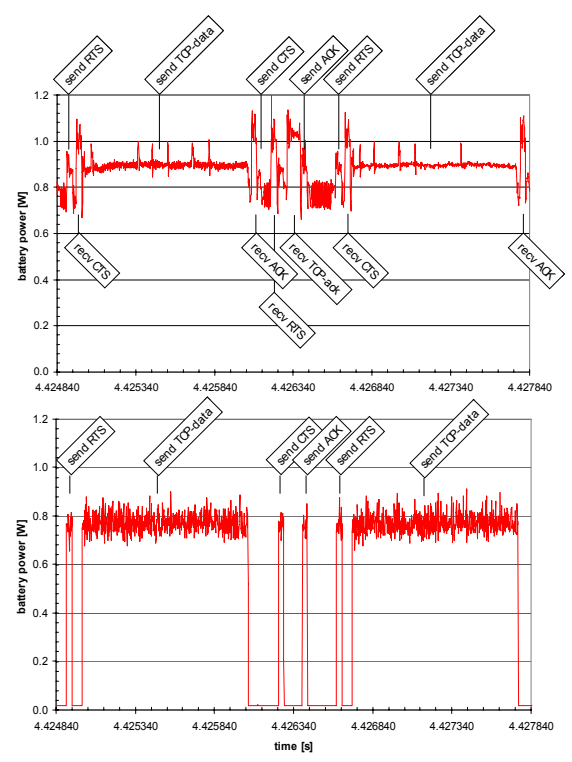

Figure 2. Power measurement samples, above ASIC and below PA. The labels point the sent and received packets.

The energy consumption of the WLAN modem is modeled by the sum of the component energies: $E_{W N I}=E_{A S I C}+E_{P A}$, where

$$
\begin{aligned}
E_{A S I C} & =\overline{P_{A S I C}} \cdot t_{s i m} \\
E_{P A} & =\sum_{i}^{n} \overline{P_{T X(i)}^{j}} \cdot t_{T X(i)}+\overline{P_{I D L E}} \cdot\left(t_{s i m}-\sum_{i}^{n} t_{T X(i)}\right) \\
t_{T X}[\mu s] & =26 \mu s+\left[\frac{22+L[b i t]}{4 \cdot R[M b p s]}\right] \cdot 4 \mu s+\Delta
\end{aligned}
$$

$\overline{P_{A S I C}}$ is the average ASIC battery power over the simulation time of $t_{s i m}, \overline{P_{T X}}$ is the average packet-specific PA battery power depending on the PHY (Physical Layer) bit rate and packet size, $\overline{P_{I D L E}}$ is the average PA leakage battery power, $t_{T X}$ is the PA activity time for processing a packet, $L$ is the packet length containing all but PLCP (Physical Layer Convergence Protocol), tail, service and pad bits and R is the PHY bit rate. In order to model $t_{T X}$ we used the analytical formula for $802.11 \mathrm{~g}$ ERP OFDM-PHY packet transmission time. However, we added a constant $\Delta=-5 \mu$ s to the original formula in order to include the impact of hardware implementation. The constant $\Delta$ is estimated from the measurements and originates from $802.11 \mathrm{~g}$ signal extension time of $6 \mu$ s (when the PA is not transmitting) and other hardware originated differences to the analytical model like ramp-up and ramp-down times, for details see [2].

The model parameters are measured with the measurement system shown in Figure 1. The heart of the hardware is the test board with WLAN modem circuitry and the host CPU running Linux OS. The test board contains measurement points that can be used for measuring the battery current drawn by the individual components on the board. The A/D converter collects battery current data with 1 $\mu$ s resolution.

\section{SIMULATION}

We compared measured and simulated energy consumption statistics in an experimental case of single file upload over fixed 12 Mbps WLAN link. For the simulation platform we used NS-2 in which we implemented the energy model. In addition, in order to simulate a mobile device, a simple model to delay packets was used (modeling of hardware originated bottlenecks, calibrated by measurements). Comparison of the simulated and measured values is shown in Table 1. As can be seen the operation and energy figures of the measurement system and the NS-2 simulator are very similar.

Table 1. Compared file upload measurement and simulation.

\begin{tabular}{|l|l|l|}
\hline \multicolumn{1}{|c|}{ Quantity } & \multicolumn{1}{c|}{ Measurement } & \multicolumn{1}{c|}{ Simulation } \\
\hline Goodput/Packets per second & $6.59 \mathrm{Mbps} / 1748$ & $6.61 \mathrm{Mbps} / 1725$ \\
Avg. PA bat.pwr./E per bit & $498 \mathrm{~mW} / 76 \mathrm{~nJ} / \mathrm{bit}$ & $498 \mathrm{~mW} / 75 \mathrm{~nJ} / \mathrm{bit}$ \\
Avg. ASIC bat.pwr./E per bit & $869 \mathrm{~mW} / 132 \mathrm{~nJ} / \mathrm{bit}$ & $869 \mathrm{~mW} / 132 \mathrm{~nJ} / \mathrm{bit}$ \\
\hline
\end{tabular}

\section{CONCLUDING REMARKS}

In this paper we have presented an energy model for WLAN 802.11g Wireless Network Interface in a Mobile handset. A new aspect in this approach is that the Power Amplifier and the Radio Modem are modeled as separated entities. The model was also implemented in NS-2 and calibrated by a measurement of a file upload case. A close match to the simulated and measured battery energies was obtained. As the next step the authors shall enhance the NS-2 802.11 MAC and PHY layers by adding link adaptation and renewing the radio environment model for more realistic simulation scenarios. This enhanced tool shall be used for the analysis of energy efficiency of the two TCP start-up algorithms: the traditional Slow-Start and challenger approach Quick-Start. Also ASIC energy model shall be improved so that it is no longer limited in the bulk file transfer use case. This requires a capability to trace operating states of the PHY. Finally, we shall introduce a model for the host CPU energy consumption.

\section{ACKNOWLEDGEMENT}

This work was supported by TEKES as part of the Future Internet program of TIVIT (Finnish Strategic Centre for Science, Technology and Innovation in the field of ICT).

\section{REFERENCES}

[1] J-P. Ebert, S. Aier, G. Kofahl, A. Becker, B. Burns and A. Wolisz. Measurement and Simulation of the Energy Consumption of an WLAN Interface. In TKN Technical Reports Series, 2002.

[2] E. Rantala, A. Karppanen, S. Granlund and P. Sarolahti. Modeling Energy Efficiency in Wireless Internet Communication. Extended version, 2009. http://www.iki.fi/pasi.sarolahti/papers/ModelingEnergyEfficie ncy-extended.pdf

[3] H. Singh and S. Singh. Energy Consumption of TCP Reno, Newreno, and SACK in Multi-Hop Wireless Networks. In Proceedings of ACM SIGMETRICS, 2002.

[4] M. Stemm and R.H. Katz. Measuring and Reducing Energy Consumption of Network Interfaces in Hand-Held Devices. In IEICE Transactions on Communications, E80-B(8):11251131, 1997. 